\title{
Lessons from Very Long-Term, Very High-Precision Photoelectric Photometry
}

\author{
G. W. Lockwood, B. A. Skiff, and D. T. Thompson \\ Lowell Observatory, Flagstaff, AZ 86001 U.S.A.
}

\begin{abstract}
Traditional photoelectric techniques can achieve millimagnitude precision if a few simple rules are scrupulously followed. We illustrate the practical limits of differential photometry obtained with a dedicated telescope and photometer. Using a highly homogeneous set of measurements of bright $F, G$, and $K$ solar analog stars, we present the distribution of observational noise and intrinsic stellar variability over a typical observing season and over the entire 8-year program. Finally, we list Strömgren uvby standards which we found to vary slightly.
\end{abstract}

\section{Introduction}

Drawing upon experience gained in a 20-year effort to monitor the brightness of solar system objects and solar analog stars as precisely as possible, we show that stellar variability can be found at millimagnitude levels over intervals ranging from days to nearly a decade. Our techniques are neither unique nor remarkable. Rather, they confirm the benefits of a straightforward, albeit steadfastly systematic, approach to differential photoelectric photometry using a dedicated reflecting telescope.

The basic procedures follow a scheme initiated by M. Jerzykiewicz in 1971, and we have found no reason to change them. In brief: (1) observe within one hour of the meridian; (2) use at least two tightly spaced comparison stars and measure them and the program star with equal weight, on the assumption that any or all may be variable; (3) complete an entire "cycle" of differential observations in one filter before moving on to another; (4) use a large star diaphragm-for our bright star program, we use 50 arc-seconds; (5) if observations don't repeat within $\sim 1 \%$, quit observing; (6) leave equipment energized and cooled year round.

\section{An example of precision differential photometry}

A program of differential, intermediate-band, Strömgren $b(472 \mathrm{~nm})$ and $y(551 \mathrm{~nm})$ photometry carried out using Lowell Observatory's $0.5-\mathrm{m}$ reflector illustrates the practical consequences of these recommendations. Since 1984, we have monitored three dozen ordinary main-sequence sunlike stars, measuring 
the rotational brightness modulations by starspots and long-term luminosity variations (e.g., Skiff and Lockwood 1985; Lockwood and Skiff 1988; Radick et al. 1990; Lockwood et al. 1992). We find detectable variability at levels as low as $0.0015 \mathrm{mag}$ rms in an 8-year time series of annual mean differential magnitudes. Conversely, the differential magnitudes of several star pairs were stable to $0.0006-0.0010 \mathrm{mag}$ rms over 8 years. Long-term measurements of planets and satellites made with the same instrumentation have produced results of similar precision over nearly two decades (Lockwood and Thompson 1991; Thompson and Lockwood 1992).

For differential observation, the stars are organized into trios or quartets, closely spaced on the sky, each containing one or two program stars and two or three comparison stars. Typically, we obtain 10-20 nights per season and four cycles of observation $(y, b, b, y)$ per night. Figure 1 is a sample light curve for two of the six differential magnitude combinations drawn from the quartet of stars featuring the program star HD82885.
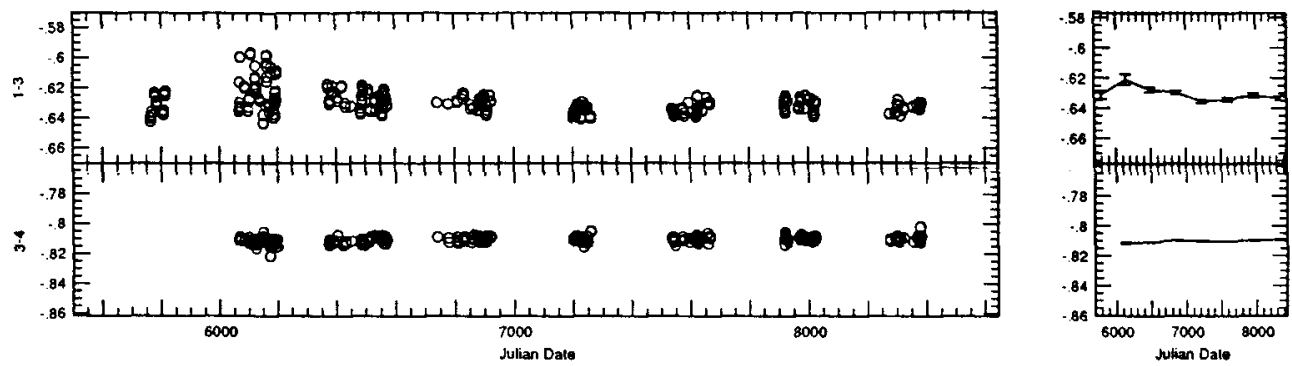

Figure 1. Differential light curves for the program star HD82885 (SV LMi, G8 IV-V, 5.4 mag, a uvby standard!) minus the comparison star HD83951 (F3 V, 6.1 mag) and, below, the comparison star HD83951 minus HD83525 (F, $7.0 \mathrm{mag}$ ). The $b$ and $y$ magnitudes are averaged in this display. Individual observations are on the left; annual mean differential magnitudes with $95 \%$ confidence interval error bars are on the right. SV LMi showed a strong rotational modulation in the second season (Skiff and Lockwood 1985) and an overall, possibly cyclic, long-term variation with an 0.015 mag peak-to-peak amplitude in the annual mean. The amplitude of variation for the comparison star pair was $0.002 \mathrm{mag}(0.0008 \mathrm{mag} \mathrm{rms})$.

The $y, b, b, y$ sequence typically requires about 35 minutes of observing time for a quartet (program star, three comparison stars). Thus, the differential magnitudes within a given $b$ or $y$ cycle are determined in less than 10 minutes; this is therefore the time interval over which sky transparency must remain constant.

\section{Variability among some $u v b y$ standard stars}

Sixteen of the more than 100 stars on our program incidentally happen to be uvby standard stars (Crawford and Barnes 1970). In the upper part of Table 1, we list ten stars that we found to be variable over the 8-year interval, 1984-1992. 
The rms deviations of their annual mean magnitudes ( $b$ and $y$ averaged) range from $0.0015-0.0053 \mathrm{mag}$. Seven of the ten are persistently variable, year after year, on a timescale of days to weeks. The remaining six, in the bottom section of the table, display fluctuations from $0.0015-0.0029 \mathrm{mag}$ rms but we were unable to detect variability. HD117176 and HD111812 are, however, variable on a seasonal timescale.

We determine the variability or constancy of a given time series of differential magnitudes according to the significance ( $99 \%$ or better) of the correlation between the magnitudes in pairs of light curves having one star in common. For example, among the six combinations of stars within a quartet group, if the light curves for star 1 minus star 2 and star 1 minus star 3 are correlated, we presume star 1 to be variable. Often, more than one such combination of light curves is available, in which case we require a consistent statistical conclusion. Thus, our determination of variability is more likely to err on the side of declaring a star to be constant when in fact it may be slightly variable. Typically, short-term (nightto-night) variability repeats at about the same level year after year and accompanies long-term variations.

Table 1. Observed Variability of Strömgren $u v b y$ Standard Stars

Standard stars which are long-term variable stars

\begin{tabular}{|c|c|c|c|c|c|c|}
\hline HD & HR & Name & Sp. Type & rms 8-yrs & rms-season & Notes \\
\hline 10476 & 493 & 107 Psc & $\mathrm{K} 1 \mathrm{~V}$ & 0.0015 & 0.0017 (const) & NSV $600^{\circ}$ \\
\hline 114710 & 4983 & $\beta$ Com & F9.5V & .0020 & .0021 (const) & \\
\hline 201092 & 8086 & 61 Cyg B & $\mathrm{K} 7 \mathrm{~V}$ & .0021 & .0021 (var) & NSV $13546^{*}$ \\
\hline 101606 & 4501 & $62 \mathrm{UMa}$ & F4V & .0021 & .0018 (var) & \\
\hline 82635 & 3800 & $10 \mathrm{LMi}$ & G7.5III & .0021 & .0049 (var) & SU LMi \\
\hline 201091 & 8085 & 61 Cyg A & K5V & .0022 & .0022 (var) & V1803 Cyg \\
\hline 115383 & 5011 & $59 \mathrm{Vir}$ & GoVs & .0033 & .0033 (var) & \\
\hline 81997 & 3759 & $\tau^{1} \mathrm{Hya}$ & F6V & .0033 & .0025 (const) & \\
\hline 39587 & 2047 & $\chi^{1}$ Ori & GOV & .0053 & .0053 (var) & \\
\hline 82885 & 3815 & $11 \mathrm{LMi}$ & G8 IV-V & .0053 & .0053 (var) & SV LMi \\
\hline
\end{tabular}

* these stars are variable, but not for the reasons given in the NSV catalog

Standard stars which appear to be constant

\begin{tabular}{|c|c|c|c|c|c|c|}
\hline HD & HR & Name & Sp. Type & rms 8-yrs & rms-season & Notes \\
\hline 143761 & 5968 & $\rho \mathrm{CrB}$ & $\mathrm{G} 0+\mathrm{Va}$ & 0.0015 & 0.0015 (const) & \\
\hline 103095 & 4550 & Grmb 1830 & G8 VI & .0017 & .0015 (const) & \\
\hline 13421 & 635 & 64 Cet & GOV & .0020 & .0020 (const) & \\
\hline 185144 & 7462 & $\sigma$ Dra & Go V & .0020 & .0020 (const) & \\
\hline 117176 & 5072 & $70 \mathrm{Vir}$ & G4 V & .0024 & .0018 (var) & \\
\hline 111812 & 4883 & $31 \mathrm{Com}$ & G0 IIIp & .0029 & .0030 (var) & \\
\hline
\end{tabular}




\section{Detection of variability on seasonal and longer time scales}

Using the correlation properties of pairs of light curves as a basis for variability assessment--whether within a single season or over much longer time intervals-we find that we can usually identify variable stars down to the level of several millimagnitudes without ambiguity unless a majority of stars in a group are variable at about the same level. In this section we describe the properties of our data.

Within the brief interval of a few minutes required to make a differential measurement, the dispersion of observed values is dominated by photon noise, scintillation noise, and, depending on the quality of the night, an additional error due to variations of sky transparency. From a very large set of homogeneous data on bright stars obtained over several years (cf. Lockwood and Skiff 1988), we show in Figure 2 the distribution of cycle-to-cycle repeatability of $b$ and $y$ differential magnitudes (that is, how well measurements repeat over a time interval of about ten minutes). The distribution is strongly skewed to higher values, probably due to the failure to discard a few marginal nights. While the median values are nearly identical for $b$ and $y, 0.0022 \mathrm{mag}$ rms and $0.0024 \mathrm{mag} \mathrm{rms}$, respectively, the small difference is statistically significant. This may be caused by inadvertently including faint red background stars in the sky measurements. Alternatively, it may be due to transparency fluctuations during the $y, b, b, y$ sequence of observation, but we suspect not.

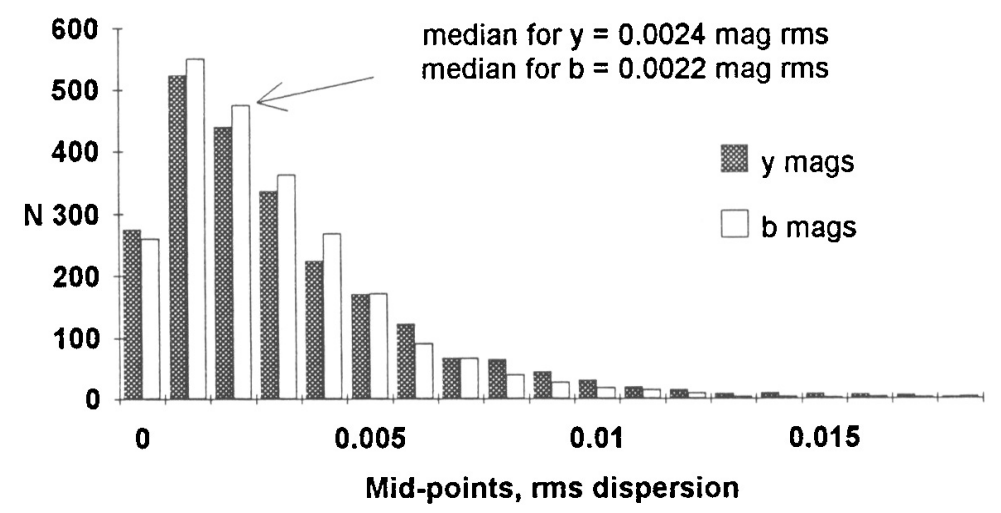

Figure 2. Distribution of cycle-to-cycle repeatability of $b$ and $y$ measurements made in $a y, b, b, y$ sequence. Values larger than about $0.005 \mathrm{mag}$ are probably caused by transparency variations, but the difference in the medians for the two filters may not be (see text).

Over the longer time intervals present in an observing season, intrinsic stellar variability begins to emerge and is generally distinguishable from observational noise on the basis of the correlations available within trios and quartets of stars. Intrinsic stellar variations are slightly greater in $b$ than in $y$, sure evidence of an astrophysical rather than an observational effect. Figure 3 shows the distribution 
of observed night-to-night variation of variable and non-variable $F, G$, and $K$, mostly main sequence, bright stars. Observational error is, of course, included in these data, dominating the distribution of values for the non-variable stars. The overlap between non-variable and variable stars is admittedly a region of some uncertainty, but it is fairly safe to say that an observed dispersion greater than 0.005 mag indicates intrinsic stellar variability.

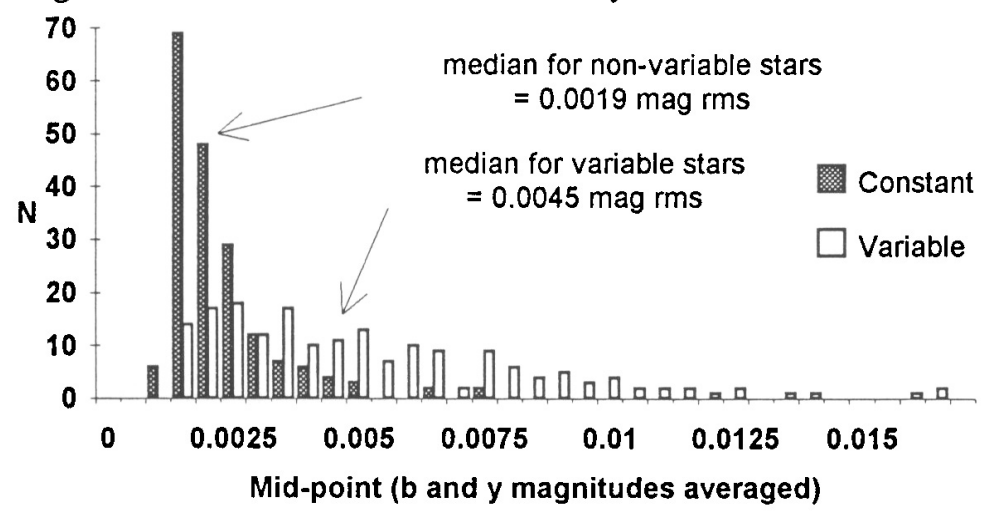

Figure 3. Distribution of the observed magnitude dispersion over an observing season for nonvariable and variable stars.

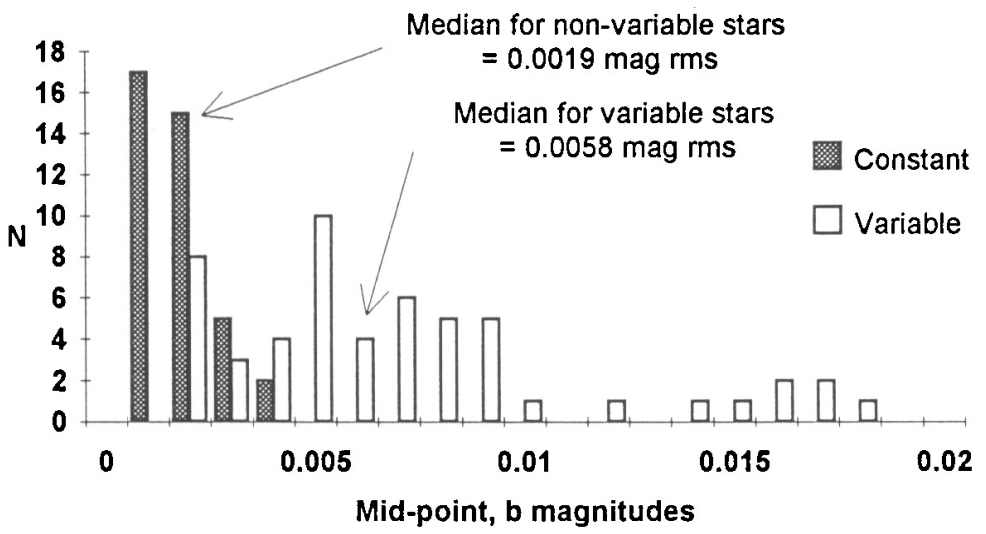

Figure 4. Distribution of the dispersion of annual mean $b$ magnitudes over 8 seasons for nonvariable and variable stars.

Figure 4 shows, similarly, the observed distribution of rms fluctuations for annual mean $b$ magnitudes, with a similar distinction between non-variable and variable stars. In this particular sample of stars, the median observed variability is about three times larger than the observational noise.

The small but astrophysically significant difference between the amount of variability in $b$ and $y$ is revealed in Figure 5, where we show the distribution of differences between the two filters. Here the non-variable stars center on zero, 
indicative of color-independent observational error, while the variable stars are displaced toward an excess of $b$ variability relative to $y$ variability.

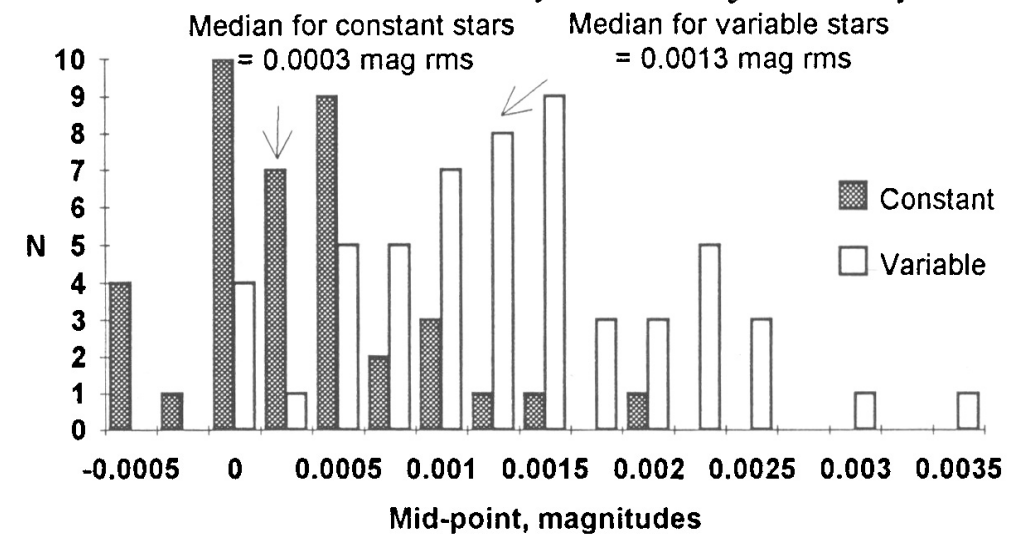

Figure 5. Distribution of the differences between the rms variability in $b$ and the rms variability in $y$. The distribution for the non-variable stars centers on zero, as expected for a dispersion arising mainly from observational error.

\section{Conclusions}

Careful, systematic differential photometry can be used to study the variability of bright stars over time intervals from days to many years down to the level of a few millimagnitudes. At this level of precision, a substantial fraction of ordinary solar analog dwarf stars, including some well-known photometric standard stars, exhibit variability. We are continuing to investigate variability in hopes of determining whether cyclic luminosity cycles are a common feature of cool dwarf stars.

The Division of Atmospheric Sciences of the National Science Foundation and the Lowell Observatory have steadfastly supported this work since 1972.

\section{References:}

Crawford, D. L., Barnes, J. V. , 1970, Astron. J. 75, 978.

Lockwood, G. W., Skiff, B. A., 1988, Luminosity Variations of Stars Similar to the Sun, Final Report, AFGL-TR-88-0221, Air Force Geophysics Lab, Hanscom Air Force Base.

Lockwood, G. W., Skiff, B. A., Baliunas, S. L., Radick, R. R., 1992, submitted to Nature.

Lockwood, G. W., Thompson, D. T., 1991, Nature 349, 593.

Radick, R. R., Lockwood, G. W., Baliunas, S. L. ,1990, Science 247, 39.

Skiff, B. A., Lockwood, G. W. ,1985, Publ. Astron. Soc. Pacific 97, 904.

Thompson, D. T., Lockwood, G. W., 1992, JGR Planets, in press. 


\section{Discussion}

R.M. Genet: Do you control the temperature of the filters? Have you seen filter aging effects?

Lockwood: No we've looked for temperature effects (nightly, seasonal) but have found none. We see a slow change in colour terms over 20 years, but since it occurs in both filters we believe it's due to the aging of the photomultipliers (an EMI $6256 \mathrm{~s}$ tube).

D.S. Hall: How often do you determine your transformation coefficients: nightly, seasonally, annually or what? I ask because transformation coefficients can be affected directly by temperature and so can be used to compensate for temperature change.

Lockwood: We compute transformation second-order coefficients in batches of 10 nights or so. However we've not noticed a seasonal variation related to temperature effects, (probably because the colour differences in our differential groups are small). The colour range of our standard stars is from $(b-y) \sim 0.0$ to $(b-y) \sim 0.4$; the color difference in differential pairs seldom exceeds half this range, i.e $\Delta(b-y)<0.2$.

E.F. Milone: CF UMa is not a variable star?

Lockwood: We've not been able to confirm its variability.

E.F. Milone: How do you maintain the stability of your PMT power supply and of PMT cooling?

Lockwood: The PMT is thermo-electrically cooled and maintained at $-15^{\circ} \mathrm{C}$. The HV power supply is never turned off and we don't know how stable it is. We do observe a small change in absolute sensitivity of the photomultipliers in the first hour or so each night as the equipment responds to changing ambient temperature. This amounts to $\sim 1 \%$. We suspect a change (drift) in the HV or the amplifier/discriminator gain, and/or band-width.

A.J. Penny: Has the reflectivity of the telescope mirror changed?

Lockwood: Yes - when we aluminized it in the mid-eighties, (probably for the first time since Harold Johnson used the telescope in the $50 \mathrm{~s}$ ), we gained $30 \%$ more photons. 